\title{
The role of simulation in the development of technical competence during surgical training: a literature review
}

\author{
Matthew P. Thomas \\ Department of Vascular Surgery, James Cook University Hospital, Marton Road, Middlesbrough, United Kingdom \\ Correspondence: Mr Matthew Thomas, 11 Herons Court, Gilesgate, Durham, DH1 2HD, United Kingdom. \\ Email: m.thomas2@nhs.net
}

Accepted: March 09, 2013

\begin{abstract}
Objectives: To establish the current state of knowledge on the effect of surgical simulation on the development of technical competence during surgical training.

Methods: Using a defined search strategy, the medical and educational literature was searched to identify empirical research that uses simulation as an educational intervention with surgical trainees. Included studies were analysed according to guidelines adapted from a Best Evidence in Medical Education review.

Results: A total of 32 studies were analysed, across 5 main categories of surgical simulation technique - use of bench models and box trainers (9 studies); Virtual Reality (14 studies); human cadavers (4 studies); animal models (2 studies) and robotics (3 studies). An improvement in technical skill was seen within the simulated environment across all five categories. This improvement was seen to
\end{abstract}

transfer to the real patient in the operating room in all categories except the use of animals.

Conclusions: Based on current evidence, surgical trainees should be confident in the effects of using simulation, and should have access to formal, structured simulation as part of their training. Surgical simulation should incorporate the use of bench models and box trainers, with the use of Virtual Reality where resources allow. Alternatives to cadaveric and animal models should be considered due to the ethical and moral issues surrounding their use, and due to their equivalency with other simulation techniques. However, any use of surgical simulation must be tailored to the individual needs of trainees, and should be accompanied by feedback from expert tutors.

Keywords: Simulation, surgical training, virtual reality, box trainers, robotics

\section{Introduction}

Surgical training has traditionally been modelled on an apprenticeship system, where trainees learn by direct instruction from their seniors, combined with long-term observation and assessment from those same seniors. This is accompanied by "the gradual absorption in to a 'community of practice' [where] participants learn as much from their peers". ${ }^{1}$ However, this traditional model has seen significant changes in recent years, driven by the European Working Time Directive (EWTD), a piece of European-wide legislation that as of August 1st 2009, introduced a maximum 48hour working week for most doctors-in-training, including trainee surgeons. ${ }^{2}$

It has been estimated that consultant surgeons have previously reached their high level of expertise after 30,000 hours of "on-the-job" training, gained through the tradi-

tional apprenticeship approach; post-EWTD, this has been revised to just 6000 hours. ${ }^{3}$ Such a reduction in hours has led to an obvious decrease in training opportunities in the operating theatre - in one region of the UK, it has been estimated that training operations have been reduced to less than a third of the minimum recommended number, and that providing trainees with the requisite training operations would require an extra 270 theatre days a year at a cost of $£ 1.3$ million. ${ }^{4}$ Such changes to working hours are therefore of significant concern to those responsible for the training of surgeons, and to trainees themselves, and have led to the search for effective methods of increasing technical skills that can be delivered outside of the operating theatre. Surgical simulation is one such method. Simulation describes "the technique of imitating the behaviour of some

48

() 2013 Matthew P Thomas. This is an Open Access article distributed under the terms of the Creative Commons Attribution License which permits unrestricted use of work provided the original work is properly cited. http://creativecommons.org/licenses/by/3.0 
situation or process.....by means of a suitably analogous situation or apparatus". ${ }^{5}$ In addition to the EWTD, there are also other significant drivers to the use of simulation in surgical training. Concerns about patient safety and the need to significantly reduce avoidable medical errors have created what has been called by some an "ethical imperative" for the use of simulation in medical education, where "patients are to be protected whenever possible......they are not commodities to be used as conveniences of training". ${ }^{6}$ Simulation away from patients and the clinical environment allows technical procedures to be broken down in to smaller, component parts that can be practiced repeatedly. This can be done at the trainees own pace, where instant feedback can be provided, both self-feedback and from senior experts. Surgical simulation can also provide training opportunities that are immediate, without having to wait for a particular "real-life" case or pathology to present itself. In a field where technological developments are so rapid, simulation also allows exposure to these new technologies and techniques early in the training period. There is also a cost implication to the use of simulation. With the use of simulation outside of the operating theatre environment, one study suggests a possible saving of $\$ 160,000$ US during training, due to faster completion of tasks, fewer errors and reduced equipment spoilage costs, plus savings in instructor/teacher costs. ${ }^{7}$ Such considerations have led to the proposal in the US of the need for a national consortium to promote the development of a national simulation system for residency training. ${ }^{8}$

There are several different surgical simulation techniques in widespread use, ranging from low-fidelity simple synthetic jigs and box-trainers to higher fidelity animal and cadaver models, through to advanced virtual reality technology. Fidelity within simulation refers to its "exactness of duplication;" in other words, the level of "realism" the simulation technique achieves. High fidelity simulation immerses the user in a more realistic and interactive environment, whereas low fidelity models use materials and equipment that are less similar to those that are actually encountered in the true operating room. ${ }^{9}$ The importance of simulation fidelity in the transfer of learning has been addressed in a recent review. High fidelity simulation was found to show clear gains in performance and transfer to the real patient setting, when compared with "typical opportunistic instruction". However, when compared to low fidelity simulation, these gains were "more modest" and in most studies, did not achieve statistically significance. It should be noted that this review did not exclusively examine simulation in surgical training, but also evaluated simulation in auscultation skills and in complex crisis management skills as well. ${ }^{10}$

The use of simulation itself is not without its disadvantages. If skills learnt during simulation are not practiced regularly thereafter, they may be rapidly lost, but without the practitioner being aware that such a loss has taken place. ${ }^{1}$ Simulation may also become "an end in itself, disconnected from the professional practice for which it purports to be a preparation". The repeated practice on "inanimate" simulation devices can also remove the human interaction that is so important in clinical practice. ${ }^{11}$

Ultimately, the role of the surgeon is to be able to safely and effectively perform operative techniques and procedures on actual patients, and with the introduction of the EWTD it is the opportunity to practice these techniques in the operating theatre that is directly affected. Both trainees, and those that train them, therefore need to be assured that the use of surgical simulation outside of the operating theatre is a valuable training tool in increasing and developing these technical skills.

Therefore, in light of its already extensive use within surgical training, both actual and proposed, and with so many professional bodies advocating its continued widespread use, this review will seek to explore the literature to ask "does the use of surgical simulation make a measurable impact on the development of technical competence in surgical training?" The objectives are:

- To synthesise the current literature on the effectiveness of these techniques in developing and improving technical competence:

i. Within the simulated environment

ii. On transfer to the real surgical patient

- To make recommendations to clinical educators on the effective use of simulation in surgical training, based on this synthesis

\section{Methods}

This research project was conducted as a literature review; formal ethical approval was therefore not required. The following databases were searched, chosen to provide the broadest range of research within the fields of healthcare and educational research: British Nursing Index (1985 to date); CINAHL (1981 to date); EMBASE (1980 to date); Medline (1950 to date); The Cochrane Library (consisting of the Cochrane Database of Systematic Reviews, Cochrane Central Register of Controlled Trials (CENTRAL), Cochrane Methodology Register, Database of Abstracts of Reviews of Effects (DARE), Health Technology Assessment Database and the NHS Economic Evaluation Database); HMIC (covering the Department of Health Library \& Information Service, 1983 to date, and the Kings Fund Information \& Library Service, 1979 to date); BREI (1975 to date); EPPI-Centre Database of Educational Research; CERUK (2000 to date); OpenGrey database (1980 to date) and the Conference Papers Index (1982 to date).

These databases were searched using the following keywords and Boolean combinations: surgical AND training; surg $^{*}$ AND simulat ${ }^{\star}$ (use of wildcard * symbol to find surgery/surgical, and simulation/simulator); technical AND skills; technical AND competence; "virtual reality" (use of “ 
" to find exact term); cadaver ${ }^{\star}$ (use of wildcard ${ }^{*}$ symbol to find cadaver/cadaveric); robot $^{*}$ AND simulat* (use of wildcard ${ }^{\star}$ symbol to find robot/robots/robotic, and simulation/simulator); animal AND simulat* (use of wildcard * symbol to find simulation/simulator). The following thesaurus terms for the above keywords were also used: "computer simulation"; "computer-assisted instruction"; "patient simulation".

The search was conducted looking for English language articles, and the title and abstract of articles identified by the above strategy were then screened. Articles were finally included where they described empirical, comparative research which utilised simulation techniques as an educational intervention. In order to keep the review as broad and extensive as possible, studies were included from any of the 9 recognised major surgical specialties (Cardiothoracic Surgery, General Surgery, Neurosurgery, Oral and Maxillofacial surgery (OMFS), Otolaryngology (ENT), Paediatric surgery, Plastic surgery, Trauma and Orthopaedic Surgery (T\&O) and Urology), and also studies involving Obstetrics \& Gynaecology trainees, a specialty which also necessitates training in surgical skills. "Citation tracking" was used to review the reference lists of all those articles meeting these criteria, in order to identify further potentially relevant work.

The focus of the review is the use of simulation in surgical training. Surgical training itself begins once medical school is completed, after a certain generic level of basic clinical competence has already been reached. Therefore all literature related to the use of surgical simulation techniques with those surgical trainees who have chosen to embark on a surgical career and started their surgical training has been included in the review. Literature focussing solely on the use of surgical simulation techniques in medical students was excluded. For the same reasons, the use of simulation solely in those who have completed their surgical training (e.g. the "expert surgeon", such as surgical consultants) was also excluded. Studies comparing the experience of surgical trainees with medical students and/or "expert" surgeons have been included where they presented data on the surgical trainees as a separate participating group. In order to determine this, studies must have clearly defined the level of experience/training of the participants; where no clear definition of this level was found, the study was excluded.

All studies meeting the final inclusion criteria were initially classified by the type of surgical simulation technique used, in order to categorise the available types of surgical simulation and to allow studies detailing similar surgical simulation techniques to be grouped together. Once grouped, studies were then further analysed following a system based on guidelines for educational studies involving simulators produced by the BEME Collaboration. ${ }^{12}$ Data was collected from this analysis on a data extraction sheet. The assessment of study design for each study was then summarised using the data extraction sheets, under the following headings: study authors/year; methodology; participants; intervention (simulation task used); outcome measures; method of evaluation (including pre-intervention measurement); results and evidence of transfer to clinical environment. A single author (MT) was responsible for the initial literature search, the screening of article titles and abstracts and the decision to finally include each article. The same author was also solely responsible for the data extraction and analysis.

\section{Results}

A total of 74 studies were identified using surgical simulation as an educational intervention. When full inclusion and exclusion criteria were applied, a final total of 32 studies qualified for full analysis. These studies fell across five main categories of surgical simulation technique - use of bench models and box trainers; Virtual Reality; animal models; human cadavers and robotics. The main study characteristics are summarised in Table 1.

\section{Bench models and box trainers}

Nine studies were included that detail the use of bench models and box-trainers; 8 studies were randomisedcontrolled trials, ${ }^{13-19,21}$ with 1 cohort study. ${ }^{20}$ Bench models are simulators that are static, and can be placed on the "bench" in front of the trainee. They use a wide variety of materials that allow the practice of skills such as knot tying, suturing (e.g. wound closure) or anastomoses (the joining of two structures, e.g. re-joining a segment of bowel to bowel, or joining blood vessels together). They are considered low-fidelity, as the materials used can be as simple as pieces of string, beads, metal hooks or stretched elastic bands. Box-trainers are used to simulate laparoscopic ("keyhole") surgery. The "box" usually has slits in its surface through which surgical instruments, including the laparoscopic camera, can be inserted. The trainee can then use the surgical instruments to manipulate materials placed inside the box. These materials can be as simple as the bench models described above, or of higher fidelity by using animal tissues. This category contained the only study to conclude that the improvement seen in operative skill was independent of simulation training. ${ }^{17}$

\section{Virtual reality}

Fourteen studies meeting the inclusion criteria were found. 11 of these were randomised-controlled trials, ${ }^{22-24,26-30,33-35}$ with 3 cohort studies. ${ }^{25,31,33}$ Three of the randomisedcontrolled trials purport to be "double-blinded", 23,26,27 however, whilst those performing the final evaluation are blinded in each study, it is impossible to blind the participants themselves to which intervention group they are in. Participant blinding would be necessary for it to be considered a double-blinded study. VR simulation describes the interaction between the trainee and a three-dimensional (3D), computer generated environment. 
Table 1. Summary of study characteristics for included studies describing empirical research involving simulation in surgical trainees

\begin{tabular}{|c|c|c|c|c|c|c|c|}
\hline Study & Methodology & Participants & $\begin{array}{c}\text { Intervention } \\
\text { (simulation task) }\end{array}$ & $\begin{array}{l}\text { Outcome } \\
\text { measures }\end{array}$ & Method of evaluation & Results & $\begin{array}{l}\text { Evidence of } \\
\text { transfer to } \\
\text { clinical } \\
\text { environment? }\end{array}$ \\
\hline $\begin{array}{l}\text { Derossis } \\
\text { et al, } 1998^{13}\end{array}$ & $\begin{array}{l}\text { Randomised } \\
\text { control trial; } \\
\text { prospective }\end{array}$ & $\begin{array}{l}12 \text { Surgical } \\
\text { residents, all } \\
\text { PGY3 }^{\star}\end{array}$ & $\begin{array}{l}5 \text { weekly practice } \\
\text { sessions on inani- } \\
\text { mate box trainer vs } \\
\text { no simulation } \\
\text { practice }\end{array}$ & $\begin{array}{l}\text { Task completion } \\
\text { time; "precision } \\
\text { of performance" } \\
\text { score; overall } \\
\text { score based on } \\
\text { the above }\end{array}$ & $\begin{array}{l}\text { Evaluation on } 7 \text { tasks } \\
\text { on box trainer, before } \\
\text { and after intervention }\end{array}$ & $\begin{array}{l}\text { Significant improvement for } \\
\text { all } 7 \text { tasks and total score } \\
\text { practice group; significant } \\
\text { improvement } 4 \text { out of } 7 \\
\text { tasks and total score no } \\
\text { practice group; improvement } \\
\text { significantly greater in } \\
\text { practice group }\end{array}$ & No \\
\hline $\begin{array}{l}\text { Scott et al, } \\
2000^{14}\end{array}$ & $\begin{array}{l}\text { Single-blind, } \\
\text { randomised } \\
\text { control trial; } \\
\text { prospective }\end{array}$ & $\begin{array}{l}27 \text { Surgical } \\
\text { residents } \\
\text { enrolled, } 22 \\
\text { completed, } \\
\text { all PGY2 \& } 3\end{array}$ & $\begin{array}{l}\text { Participation in } \\
\text { training curriculum on } \\
\text { box trainer vs no } \\
\text { simulation training }\end{array}$ & $\begin{array}{l}\text { Task completion } \\
\text { time; global } \\
\text { assessment } \\
\text { score covering } 8 \\
\text { components of } \\
\text { technical } \\
\text { procedure }\end{array}$ & $\begin{array}{l}\text { Evaluation on } 5 \text { tasks } \\
\text { on box trainer and } \\
\text { performance of } \\
\text { operative procedure } \\
\text { on real patient, before } \\
\text { and after intervention }\end{array}$ & $\begin{array}{l}\text { Significantly greater } \\
\text { reduction in task completion } \\
\text { time in training group; } \\
\text { significantly greater } \\
\text { improvement in } 4 \text { out of } 8 \\
\text { aspects global assessment } \\
\text { score in training group }\end{array}$ & Yes \\
\hline $\begin{array}{l}\text { Hamilton } \\
\text { et al, } 2001^{15}\end{array}$ & $\begin{array}{l}\text { Single-blind, } \\
\text { randomised } \\
\text { control trial; } \\
\text { prospective }\end{array}$ & $\begin{array}{l}21 \text { Surgical } \\
\text { residents, all } \\
\text { PGY3 \& } 4\end{array}$ & $\begin{array}{l}\text { Participation in } \\
\text { training curriculum on } \\
\text { box trainer vs. no } \\
\text { simulation training }\end{array}$ & $\begin{array}{l}\text { Global assess- } \\
\text { ment score } \\
\text { covering } 8 \\
\text { components of } \\
\text { technical } \\
\text { procedure, } \\
\text { including } \\
\text { composite score }\end{array}$ & $\begin{array}{l}\text { Performance of } \\
\text { operative procedure } \\
\text { on real patient in } \\
\text { operating room, } \\
\text { before and after } \\
\text { intervention }\end{array}$ & $\begin{array}{l}\text { Significantly higher compo- } \\
\text { site score in } 5 \text { out of } 8 \\
\text { components of global } \\
\text { assessment, plus significant } \\
\text { improvement in composite } \\
\text { score and in } 4 \text { out of } 8 \\
\text { components of global } \\
\text { assessment in training } \\
\text { group }\end{array}$ & Yes \\
\hline $\begin{array}{l}\text { Risucci } \\
\text { et al, } 2001^{16}\end{array}$ & $\begin{array}{l}\text { Randomised } \\
\text { control trial; } \\
\text { prospective }\end{array}$ & $\begin{array}{l}14 \text { Surgical } \\
\text { residents, all } \\
\text { PGY1 }\end{array}$ & $\begin{array}{l}2 \text { tasks on inanimate } \\
\text { bench model } \\
\text { repeated } 9 \text { times, } \\
\text { either with or without } \\
\text { additional instruction } \\
\text { (demonstration video } \\
\text { and direct feedback) }\end{array}$ & $\begin{array}{l}\text { Task completion } \\
\text { time; total } \\
\text { number of errors }\end{array}$ & $\begin{array}{l}\text { Pre-test evaluation } \\
\text { after completing each } \\
\text { task; post-test mean } \\
\text { task completion and } \\
\text { total error score }\end{array}$ & $\begin{array}{l}\text { Task completion time } \\
\text { significantly reduced both } \\
\text { groups; error score and } \\
\text { variance in number of errors } \\
\text { significantly greater without } \\
\text { instruction }\end{array}$ & No \\
\hline $\begin{array}{l}\text { Traxer et al, } \\
2001^{17}\end{array}$ & $\begin{array}{l}\text { Single-blind, } \\
\text { randomised } \\
\text { control trial; } \\
\text { prospective }\end{array}$ & $\begin{array}{l}12 \text { Urology } \\
\text { residents, all } \\
\text { PGY3 to } 5\end{array}$ & $\begin{array}{l}10 \text { days skills training } \\
\text { on inanimate box } \\
\text { trainer vs no skills } \\
\text { training }\end{array}$ & $\begin{array}{l}\text { Task completion } \\
\text { time; perfor- } \\
\text { mance score } \\
\text { assessing } 7 \\
\text { components of } \\
\text { technical } \\
\text { procedure }\end{array}$ & $\begin{array}{l}\text { Cumulative task } \\
\text { completion time on } 5 \\
\text { tasks performed on } \\
\text { box trainer; perfor- } \\
\text { mance score on } \\
\text { operative procedure } \\
\text { performed on live } \\
\text { animal }\end{array}$ & $\begin{array}{l}\text { Significant decrease in task } \\
\text { completion time in skills } \\
\text { training group; performance } \\
\text { score significantly improved } \\
\text { in both groups }\end{array}$ & No \\
\hline $\begin{array}{l}\text { Korndorffer } \\
\text { et } a l, 2005^{18}\end{array}$ & $\begin{array}{l}\text { Single-blind, } \\
\text { randomised } \\
\text { control trial; } \\
\text { prospective }\end{array}$ & $\begin{array}{l}17 \text { Surgical } \\
\text { residents, } \\
\text { PGY1 to } 5\end{array}$ & $\begin{array}{l}8 \text { weeks practice on } \\
\text { inanimate box trainer } \\
\text { vs no simulation } \\
\text { practice }\end{array}$ & $\begin{array}{l}\text { Task completion } \\
\text { time; accuracy } \\
\text { errors; knot } \\
\text { security; overall } \\
\text { score based on } \\
\text { all the above }\end{array}$ & $\begin{array}{l}\text { Evaluation of suturing } \\
\text { task performed on } \\
\text { live animal, before } \\
\text { and after intervention }\end{array}$ & $\begin{array}{l}\text { Significant improvement in } \\
\text { task completion time, } \\
\text { accuracy errors and overall } \\
\text { score, plus significantly } \\
\text { higher completion time and } \\
\text { overall score in practice } \\
\text { group; significant improve- } \\
\text { ment in completion time and } \\
\text { overall score in no practice } \\
\text { group }\end{array}$ & Yes \\
\hline $\begin{array}{l}\text { Banks et al, } \\
2007^{19}\end{array}$ & $\begin{array}{l}\text { Single-blind, } \\
\text { randomised } \\
\text { control trial; } \\
\text { prospective }\end{array}$ & $\begin{array}{l}20 \text { Obstetrics } \\
\text { and Gynae- } \\
\text { cology } \\
\text { Residents, all } \\
\text { PGY1 }\end{array}$ & $\begin{array}{l}\text { Surgical teaching in } \\
\text { operating room vs } \\
\text { teaching in operating } \\
\text { room plus participa- } \\
\text { tion in surgical skills } \\
\text { laboratory }\end{array}$ & $\begin{array}{l}\text { Written assess- } \\
\text { ment; task- } \\
\text { specific check- } \\
\text { list; global rating } \\
\text { scale; "pass-fail" } \\
\text { rating }\end{array}$ & $\begin{array}{l}\text { Evaluation of } \\
\text { simulated procedure } \\
\text { on inanimate box } \\
\text { trainer plus assess- } \\
\text { ment of operative } \\
\text { procedure on real } \\
\text { patient, before and } \\
\text { after intervention }\end{array}$ & $\begin{array}{l}\text { Significantly higher score in } \\
\text { all assessments post- } \\
\text { intervention in the skills } \\
\text { laboratory group }\end{array}$ & Yes \\
\hline $\begin{array}{l}\text { Joyce et al, } \\
2010^{20}\end{array}$ & $\begin{array}{l}\text { Cohort study; } \\
\text { prospective; } \\
\text { no evidence of } \\
\text { randomisation }\end{array}$ & $\begin{array}{l}11 \text { Surgical } \\
\text { residents (2 } \\
\text { PGY1, } 2 \\
\text { PGY2, } 2 \\
\text { PGY3; } 2 \mathrm{~T}^{\dagger}{ }^{\dagger} \\
2 \mathrm{~T} 2,1 \mathrm{~T} 3)\end{array}$ & $\begin{array}{l}2 \text { weeks practice on } \\
\text { inanimate bench } \\
\text { model }\end{array}$ & $\begin{array}{l}\text { Task completion } \\
\text { time; Objective } \\
\text { Structured } \\
\text { Assessment of } \\
\text { Technical Skill } \\
\text { (OSAT), } \\
\text { covering } 11 \\
\text { technical } \\
\text { components of } \\
\text { procedure }\end{array}$ & $\begin{array}{l}\text { Performance of } \\
\text { procedure on ex vivo } \\
\text { animal model, before } \\
\text { and after intervention }\end{array}$ & $\begin{array}{l}\text { Significant improvement in } \\
\text { task completion time and all } \\
11 \text { component of OSAT }\end{array}$ & No \\
\hline $\begin{array}{l}\text { Price et al, } \\
2011^{21}\end{array}$ & $\begin{array}{l}\text { Single-blind, } \\
\text { randomised } \\
\text { control trial; } \\
\text { prospective }\end{array}$ & $\begin{array}{l}39 \text { Surgical } \\
\text { residents }(29 \\
\text { PGY1, } 10 \\
\text { PGY2) }\end{array}$ & $\begin{array}{l}\text { Expert tutorial vs } \\
\text { expert tutorial plus } 2 \\
\text { weeks training on } \\
\text { bench model }\end{array}$ & $\begin{array}{l}\text { Task completion } \\
\text { time; OSAT; } \\
\text { global rating } \\
\text { scale }\end{array}$ & $\begin{array}{l}\text { Pre-test score on } \\
\text { bench model; post- } \\
\text { test on live animal } \\
\text { model }\end{array}$ & $\begin{array}{l}\text { All outcome measures } \\
\text { significantly higher in training } \\
\text { group }\end{array}$ & No \\
\hline
\end{tabular}


Thomas Simulation and technical competence in surgical training

\begin{tabular}{|c|c|c|c|c|c|c|c|}
\hline Study & Methodology & Participants & $\begin{array}{c}\text { Intervention } \\
\text { (simulation task) }\end{array}$ & $\begin{array}{l}\text { Outcome } \\
\text { measures }\end{array}$ & Method of evaluation & Results & $\begin{array}{l}\text { Evidence of } \\
\text { transfer to } \\
\text { clinical } \\
\text { environment? }\end{array}$ \\
\hline $\begin{array}{l}\text { Hamilton } \\
\text { et al, } 2002^{22}\end{array}$ & $\begin{array}{l}\text { Single-blind, } \\
\text { randomised } \\
\text { control trial; } \\
\text { prospective }\end{array}$ & $\begin{array}{l}50 \text { Surgical } \\
\text { residents } \\
\text { enrolled, } 49 \\
\text { completed } \\
\text { (30 PGY1, } \\
19 \text { PGY2) }\end{array}$ & $\begin{array}{l}\text { Training on VR } \\
\text { simulator vs training } \\
\text { on inanimate box } \\
\text { trainer }\end{array}$ & $\begin{array}{l}\text { Task perfor- } \\
\text { mance score; } \\
\text { global assess- } \\
\text { ment score }\end{array}$ & $\begin{array}{l}\text { Evaluation of } 6 \text { (VR } \\
\text { group) or } 5 \text { (box trainer } \\
\text { group) tasks, before } \\
\text { and after intervention; } \\
\text { PGY2 Residents } \\
\text { evaluated on procedure } \\
\text { on real patient, before } \\
\text { and after intervention }\end{array}$ & $\begin{array}{l}\text { Task performance score } \\
\text { significantly improved in } \\
\text { both groups; global } \\
\text { assessment score on real } \\
\text { patient significantly } \\
\text { improved in VR group only }\end{array}$ & Yes \\
\hline $\begin{array}{l}\text { Seymour et } \\
a l, 2002^{23}\end{array}$ & $\begin{array}{l}\text { Double-blind, } \\
\text { randomised } \\
\text { control trial; } \\
\text { prospective }\end{array}$ & $\begin{array}{l}16 \text { Surgical } \\
\text { Residents } \\
\text { (PGY1 to 4) }\end{array}$ & $\begin{array}{l}\text { "Standard" training } \\
\text { plus training on VR } \\
\text { simulator vs } \\
\text { "standard" training } \\
\text { alone }\end{array}$ & $\begin{array}{l}\text { Task completion } \\
\text { time; total } \\
\text { number of errors }\end{array}$ & $\begin{array}{l}\text { Evaluation of operative } \\
\text { procedure on real } \\
\text { patient, after interven- } \\
\text { tion }\end{array}$ & $\begin{array}{l}\text { Statistically significant } \\
\text { improvement in error } \\
\text { number with less variability } \\
\text { in performance in VR group }\end{array}$ & Yes \\
\hline $\begin{array}{l}\text { Grantcharov } \\
\text { et al, } 2004^{24}\end{array}$ & $\begin{array}{l}\text { Single-blind, } \\
\text { randomised } \\
\text { control trial; } \\
\text { prospective }\end{array}$ & $\begin{array}{l}20 \text { Surgical } \\
\text { Trainees } \\
\text { (median } \\
\text { time from } \\
\text { graduation } 7 \\
\text { years) } \\
\end{array}$ & $\begin{array}{l}\text { Training on VR } \\
\text { simulator vs no } \\
\text { simulation training }\end{array}$ & $\begin{array}{l}\text { Task completion } \\
\text { time; error score; } \\
\text { "economy of } \\
\text { movement" } \\
\text { score }\end{array}$ & $\begin{array}{l}\text { Evaluation of operative } \\
\text { procedure on real } \\
\text { patient, before and after } \\
\text { intervention }\end{array}$ & $\begin{array}{l}\text { Significant improvement in } \\
\text { all outcome measures in VR } \\
\text { training group }\end{array}$ & Yes \\
\hline $\begin{array}{l}\text { Dayal et al, } \\
2004^{25}\end{array}$ & $\begin{array}{l}\text { Cohort study; } \\
\text { prospective; } \\
\text { no evidence of } \\
\text { randomisation }\end{array}$ & $\begin{array}{l}16 \text { Surgical } \\
\text { Residents } \\
\text { ("Novice" } \\
\text { group) + } 5 \\
\text { "Expert" } \\
\text { Surgeons }\end{array}$ & $\begin{array}{l}2 \text { hours of training on } \\
\text { VR simulator, training } \\
\text { given by "expert" }\end{array}$ & $\begin{array}{l}\text { Task-specific } \\
\text { checklist; } \\
\text { "fluoroscopy } \\
\text { time"; amount of } \\
\text { dye used; } \\
\text { subjective } \\
\text { evaluation of } \\
\text { technical ability }\end{array}$ & $\begin{array}{l}\text { Evaluation of simulated } \\
\text { operative procedure on } \\
\text { VR simulator, before } \\
\text { and after intervention }\end{array}$ & $\begin{array}{l}\text { Significant improvement in } \\
\text { all outcome measures in } \\
\text { Novice group }\end{array}$ & No \\
\hline $\begin{array}{l}\text { McClusky et } \\
\text { al, } 2004^{26}\end{array}$ & $\begin{array}{l}\text { Double-blind, } \\
\text { randomised } \\
\text { control trial; } \\
\text { prospective }^{\ddagger}\end{array}$ & $\begin{array}{l}12 \text { Surgical } \\
\text { Residents } \\
\text { (PGY } 1 \& 2 \text { ) }\end{array}$ & $\begin{array}{l}\text { Training on VR } \\
\text { simulator vs } \\
\text { "standard" training }\end{array}$ & $\begin{array}{l}\text { Task completion } \\
\text { time; error score }\end{array}$ & $\begin{array}{l}\text { Evaluation of operative } \\
\text { procedure on real } \\
\text { patient, after interven- } \\
\text { tion }\end{array}$ & $\begin{array}{l}\text { Improvement in task } \\
\text { completion time and error } \\
\text { score in VR group, no } \\
\text { evidence of significance }\end{array}$ & Yes \\
\hline $\begin{array}{l}\text { Andreatta et } \\
\text { al, } 2006^{27}\end{array}$ & $\begin{array}{l}\text { Double-blind, } \\
\text { randomised } \\
\text { control trial; } \\
\text { prospective }\end{array}$ & $\begin{array}{l}21 \text { Surgical } \\
\text { Interns } \\
\text { enrolled, } 19 \\
\text { completed }\end{array}$ & $\begin{array}{l}\text { Training on VR } \\
\text { simulator vs no } \\
\text { simulation training }\end{array}$ & $\begin{array}{l}\text { "Time and } \\
\text { accuracy" } \\
\text { assessment; } \\
\text { global assess- } \\
\text { ment score }\end{array}$ & $\begin{array}{l}\text { Evaluation of operative } \\
\text { procedure performed on } \\
\text { anaesthetised pig, after } \\
\text { intervention }\end{array}$ & $\begin{array}{l}\text { VR group performed } \\
\text { significantly better in } 5 \text { out of } \\
6 \text { parameters of global } \\
\text { assessment score }\end{array}$ & No \\
\hline $\begin{array}{l}\text { Chaer et al, } \\
2006^{28}\end{array}$ & $\begin{array}{l}\text { Single-blind, } \\
\text { randomised } \\
\text { control trial; } \\
\text { prospective }\end{array}$ & $\begin{array}{l}20 \text { Surgical } \\
\text { Residents }\end{array}$ & $\begin{array}{l}\text { Didactic training plus } \\
\text { training on VR } \\
\text { simulator vs didactic } \\
\text { training alone }\end{array}$ & $\begin{array}{l}\text { Task specific } \\
\text { checklist; global } \\
\text { assessment } \\
\text { score covering } \\
12 \text { components } \\
\text { of technical } \\
\text { procedure }\end{array}$ & $\begin{array}{l}\text { Evaluation on } 2 \\
\text { consecutive technical } \\
\text { procedures performed } \\
\text { on real patients, after } \\
\text { intervention }\end{array}$ & $\begin{array}{l}\text { Significantly better perfor- } \\
\text { mance in task specific } \\
\text { checklist and global } \\
\text { assessment score in VR } \\
\text { group }\end{array}$ & Yes \\
\hline $\begin{array}{l}\text { Ahlberg et } \\
a l, 200{ }^{29}\end{array}$ & $\begin{array}{l}\text { Single-blind, } \\
\text { randomised } \\
\text { control trial; } \\
\text { prospective }\end{array}$ & $\begin{array}{l}13 \text { Surgical } \\
\text { Residents } \\
\text { (all PGY1 \& } \\
\text { 2) }\end{array}$ & $\begin{array}{l}\text { Training on VR } \\
\text { simulator vs no } \\
\text { simulation training }\end{array}$ & $\begin{array}{l}\text { Task completion } \\
\text { time; error score }\end{array}$ & $\begin{array}{l}\text { Evaluation of operative } \\
\text { procedure performed } \\
\text { between } 5 \text { to } 10 \text { times } \\
\text { on real patients, after } \\
\text { intervention }\end{array}$ & $\begin{array}{l}\text { VR group made significantly } \\
\text { fewer errors; task comple- } \\
\text { tion time shorter in VR group } \\
\text { bit did not reach significance }\end{array}$ & Yes \\
\hline $\begin{array}{l}\text { Cosman et } \\
a l, 2007^{30}\end{array}$ & $\begin{array}{l}\text { Single-blind, } \\
\text { randomised } \\
\text { control trial; } \\
\text { prospective }\end{array}$ & $\begin{array}{l}10 \text { Surgical } \\
\text { Trainees }\end{array}$ & $\begin{array}{l}\text { Training on VR } \\
\text { simulator vs no } \\
\text { simulation training }\end{array}$ & $\begin{array}{l}\text { Task completion } \\
\text { time; error } \\
\text { assessment } \\
\text { score; "bimanual } \\
\text { co-ordination"; } \\
\text { global assess- } \\
\text { ment score }\end{array}$ & $\begin{array}{l}\text { Evaluation of operative } \\
\text { procedure performed on } \\
\text { real patient, after } \\
\text { intervention }\end{array}$ & $\begin{array}{l}\text { Significant improvement in } \\
\text { error score, bi-manual co- } \\
\text { ordination and global } \\
\text { assessment score in VR } \\
\text { group; borderline improve- } \\
\text { ment in task completion time } \\
\text { in VR group }\end{array}$ & Yes \\
\hline $\begin{array}{l}\text { Dawson et } \\
a^{3 l, 2007}{ }^{31}\end{array}$ & $\begin{array}{l}\text { Cohort study; } \\
\text { prospective; } \\
\text { no evidence of } \\
\text { randomisation }\end{array}$ & $\begin{array}{l}9 \text { Surgical } \\
\text { Residents }\end{array}$ & $\begin{array}{l}\text { Participation in "skills } \\
\text { workshop", including } \\
8 \text { hours of VR } \\
\text { simulation training }\end{array}$ & $\begin{array}{l}5 \text { procedure- } \\
\text { related metrics, } \\
\text { including task } \\
\text { completion time }\end{array}$ & $\begin{array}{l}\text { Evaluation on simulated } \\
\text { procedure, before and } \\
\text { after intervention }\end{array}$ & $\begin{array}{l}\text { Significant improvement in } 3 \\
\text { out of } 5 \text { outcome measures, } \\
\text { including task completion } \\
\text { time, fluoroscopy time and } \\
\text { amount of contrast used }\end{array}$ & No \\
\hline $\begin{array}{l}\text { Bal- } \\
\text { asundaram, } \\
\text { Aggarwal \& } \\
\text { Darzi, } 2008 \\
32\end{array}$ & $\begin{array}{l}\text { Cohort study; } \\
\text { prospective; } \\
\text { no evidence of } \\
\text { randomisation }\end{array}$ & $\begin{array}{l}10 \text { Junior } \\
\text { Surgical } \\
\text { Residents }\end{array}$ & $\begin{array}{l}\text { Repetition of } 5 \text { tasks } \\
\text { on VR simulator }\end{array}$ & $\begin{array}{l}\text { Task completion } \\
\text { time; error score; } \\
\text { instrument path- } \\
\text { length }\end{array}$ & $\begin{array}{l}\text { Evaluation over } 5 \text { tasks } \\
\text { on VR simulator, } \\
\text { repeated } 10 \text { times each }\end{array}$ & $\begin{array}{l}\text { Significant learning curve } \\
\text { seen for task completion } \\
\text { time and instrument path- } \\
\text { length, but not for error } \\
\text { score }\end{array}$ & No \\
\hline $\begin{array}{l}\text { Verdaasdon } \\
\mathrm{k} \text { et al, } 2008\end{array}$ & $\begin{array}{l}\text { Single-blind, } \\
\text { randomised } \\
\text { control trial; } \\
\text { prospective }\end{array}$ & $\begin{array}{l}20 \text { Surgical } \\
\text { Trainees (all } \\
1^{\text {st }} \text { and } 2^{\text {nd }} \\
\text { year) }\end{array}$ & $\begin{array}{l}\text { Training on VR } \\
\text { simulator vs no } \\
\text { simulation training }\end{array}$ & $\begin{array}{l}\text { Task completion } \\
\text { time; error } \\
\text { assessment } \\
\text { score; global } \\
\text { rating scale }\end{array}$ & $\begin{array}{l}\text { Evaluation of technical } \\
\text { procedure performed on } \\
\text { anaesthetised pig, after } \\
\text { intervention }\end{array}$ & $\begin{array}{l}\text { Significant improvement in } \\
\text { task completion time and } \\
\text { error score in VR group; no } \\
\text { difference found in global } \\
\text { rating scale }\end{array}$ & No \\
\hline $\begin{array}{l}\text { Larsen et al, } \\
2009^{34}\end{array}$ & $\begin{array}{l}\text { Single-blind, } \\
\text { randomised } \\
\text { control trial; } \\
\text { prospective }\end{array}$ & $\begin{array}{l}24 \text { Obstet- } \\
\text { rics \& } \\
\text { Gynaecolo- } \\
\text { gy Regis- } \\
\text { trars } \\
\text { enrolled, } 21 \\
\text { completed } \\
\text { (PGY3 to 8) }\end{array}$ & $\begin{array}{l}\text { Training on VR } \\
\text { simulator vs } \\
\text { "standard" clinical } \\
\text { training }\end{array}$ & $\begin{array}{l}\text { Task completion } \\
\text { time; perfor- } \\
\text { mance score }\end{array}$ & $\begin{array}{l}\text { Evaluation of technical } \\
\text { procedure performed on } \\
\text { real patient, after } \\
\text { intervention }\end{array}$ & $\begin{array}{l}\text { Task completion time } \\
\text { significantly shorter and } \\
\text { performance score signifi- } \\
\text { cantly higher in VR group }\end{array}$ & Yes \\
\hline
\end{tabular}




\begin{tabular}{|c|c|c|c|c|c|c|c|}
\hline Study & Methodology & Participants & $\begin{array}{c}\text { Intervention } \\
\text { (simulation task) }\end{array}$ & $\begin{array}{l}\text { Outcome } \\
\text { measures }\end{array}$ & Method of evaluation & Results & $\begin{array}{l}\text { Evidence of } \\
\text { transfer to } \\
\text { clinical } \\
\text { environment? }\end{array}$ \\
\hline $\begin{array}{l}\text { Maschuw } \\
\text { et al, } 2011^{35}\end{array}$ & $\begin{array}{l}\text { Single-blind, } \\
\text { randomised } \\
\text { control trial; } \\
\text { prospective }\end{array}$ & $\begin{array}{l}50 \text { Surgical } \\
\text { Residents } \\
\text { (all PGY1) }\end{array}$ & $\begin{array}{l}\text { Training on VR } \\
\text { simulator vs no } \\
\text { simulator training }\end{array}$ & $\begin{array}{l}\text { Task completion } \\
\text { time, "tissue } \\
\text { damage" score; } \\
\text { "economy of } \\
\text { motion" score }\end{array}$ & $\begin{array}{l}\text { Evaluation of } 7 \text { tasks } \\
\text { performed on VR } \\
\text { simulator, before and } \\
\text { after intervention }\end{array}$ & $\begin{array}{l}\text { Significant improvement in } \\
\text { all outcome measures in VR } \\
\text { group }\end{array}$ & No \\
\hline $\begin{array}{l}\text { Fried et al, } \\
1999^{36}\end{array}$ & $\begin{array}{l}\text { Randomised } \\
\text { control trial; } \\
\text { prospective }\end{array}$ & $\begin{array}{l}12 \text { Surgical } \\
\text { Residents, all } \\
\text { PGY3 }\end{array}$ & $\begin{array}{l}5 \text { weekly practice } \\
\text { sessions on inani- } \\
\text { mate box trainer, vs. } \\
\text { no practice }\end{array}$ & $\begin{array}{l}\text { Performance } \\
\text { score (PS) } \\
\text { based on task } \\
\text { completion time } \\
\text { and precision of } \\
\text { performance }\end{array}$ & $\begin{array}{l}7 \text { different operative } \\
\text { tasks, evaluated on } \\
\text { inanimate simulator and } \\
\text { anaesthetized pig } \\
\text { model, before and after } \\
\text { intervention }\end{array}$ & $\begin{array}{l}\text { PS significantly increased in } \\
\text { practice group in } 5 \text { out of } 7 \\
\text { tasks, vs } 1 \text { out of } 7 \text { task for } \\
\text { no practice group }\end{array}$ & No \\
\hline $\begin{array}{l}\text { Bijoy } \\
\text { Thomas } \\
\text { et al, } 2010^{37}\end{array}$ & $\begin{array}{l}\text { Cohort study; } \\
\text { prospective; } \\
\text { no evidence } \\
\text { of randomisa- } \\
\text { tion }\end{array}$ & $\begin{array}{l}31 \text { Obstetrics } \\
\text { \& Gynaecol- } \\
\text { ogy Resi- } \\
\text { dents - } 7 \\
\text { PGY1, } 8 \\
\text { PGY2, 11 } \\
\text { PGY3, } 5 \\
\text { PGY4 }\end{array}$ & $\begin{array}{l}1 \text { hour practice } \\
\text { session under direct } \\
\text { supervision, further } \\
1-2 \text { hours practice } \\
\text { session unsuper- } \\
\text { vised, using ex vivo } \\
\text { porcine model }\end{array}$ & $\begin{array}{l}\text { Subjective self- } \\
\text { assessment } \\
\text { questionnaire } \\
\text { using 10-point } \\
\text { Likert Scale }\end{array}$ & $\begin{array}{l}\text { Pre and post- } \\
\text { intervention self- } \\
\text { assessment question- } \\
\text { naire }\end{array}$ & $\begin{array}{l}\text { Significant improvement in } \\
\text { self-perception of comfort } \\
\text { level and knowledge of } \\
\text { procedure, and of familiarity } \\
\text { with surgical instruments }\end{array}$ & No \\
\hline $\begin{array}{l}\text { Martin et al, } \\
1998^{38}\end{array}$ & $\begin{array}{l}\text { Cohort study; } \\
\text { prospective; } \\
\text { no evidence } \\
\text { of randomisa- } \\
\text { tion }\end{array}$ & $\begin{array}{l}8 \text { Surgical } \\
\text { Residents, all } \\
\text { PGY1 }{ }^{1}\end{array}$ & $\begin{array}{l}\text { Cadaveric laboratory, } \\
\text { practising } 3 \text { technical } \\
\text { procedures on } 2 \\
\text { occasions } 3 \text { weeks } \\
\text { apart }\end{array}$ & $\begin{array}{l}\text { Task completion } \\
\text { time and number } \\
\text { of complications }\end{array}$ & $\begin{array}{l}\text { Evaluation of } 3 \\
\text { procedures on cadaver, } \\
\text { immediately post- } \\
\text { instruction \& } 3 \text { weeks } \\
\text { later }\end{array}$ & $\begin{array}{l}\text { Completion time }+ \text { no. of } \\
\text { complications. decreased } \\
\text { significantly, both post- } \\
\text { instruction }+3 \text { weeks later }\end{array}$ & $\begin{array}{l}\text { Yes - partici- } \\
\text { pants evaluat- } \\
\text { ed on real } \\
\text { patients } \\
\text { following } \\
\text { completion of } \\
\text { cadaveric } \\
\text { laboratory } \\
\text { training }\end{array}$ \\
\hline $\begin{array}{l}\text { Anastakis } \\
\text { et al, } 1999{ }^{39}\end{array}$ & $\begin{array}{l}\text { Single-blind, } \\
\text { randomised } \\
\text { control trial; } \\
\text { prospective }\end{array}$ & $\begin{array}{l}23 \text { Surgical } \\
\text { Residents, all } \\
\text { PGY1 }\end{array}$ & $\begin{array}{l}\text { Training on human } \\
\text { cadaver vs. training } \\
\text { on inanimate bench } \\
\text { model vs. learning } \\
\text { from prepared text } \\
\text { only }\end{array}$ & $\begin{array}{l}\text { Task-specific } \\
\text { checklist and } \\
\text { global rating } \\
\text { scale of } \\
\text { operative skill }\end{array}$ & $\begin{array}{l}\text { Performance of } 6 \\
\text { different operative } \\
\text { procedures on human } \\
\text { cadaver; no pre- } \\
\text { intervention measure- } \\
\text { ment }\end{array}$ & $\begin{array}{l}\text { Checklist \& global score } \\
\text { significantly higher after } \\
\text { bench and cadaver training; } \\
\text { results of bench and } \\
\text { cadaver training equivalent }\end{array}$ & No \\
\hline $\begin{array}{l}\text { Bergeson } \\
\text { et al, } 2008{ }^{40}\end{array}$ & $\begin{array}{l}\text { Cohort study; } \\
\text { prospective; } \\
\text { no evidence } \\
\text { of randomisa- } \\
\text { tion }\end{array}$ & $\begin{array}{l}3 \text { Orthopae- } \\
\text { dic Residents } \\
\text { - } 2 \text { PGY1, } 1 \\
\text { PGY3 }\end{array}$ & $\begin{array}{l}\text { Use of vertebral body } \\
\text { from cadaveric } \\
\text { thoracic spines }\end{array}$ & $\begin{array}{l}\text { Error rate, } \\
\text { "critical" error } \\
\text { rate", error } \\
\text { awareness rate }\end{array}$ & $\begin{array}{l}\text { Consecutive instrumen- } \\
\text { tation of vertebral } \\
\text { bodies from } 5 \text { cadaveric } \\
\text { spine }\end{array}$ & $\begin{array}{l}\text { Error rate significantly } \\
\text { decreased } 3^{\text {rd }} \text { to } 5^{\text {th }} \text { spines, } \\
\text { "critical" error rate signifi- } \\
\text { cantly decreased } 4^{\text {th }} \& 5^{\text {th }} \\
\text { spines }\end{array}$ & No \\
\hline $\begin{array}{l}\text { Martin et al, } \\
2011^{41}\end{array}$ & $\begin{array}{l}\text { Cohort study; } \\
\text { prospective; } \\
\text { no evidence } \\
\text { of randomisa- } \\
\text { tion }\end{array}$ & $\begin{array}{l}15 \text { Ortho- } \\
\text { paedic } \\
\text { Residents + } \\
4 \text { Attending } \\
\text { Surgeons }\end{array}$ & $\begin{array}{l}3 \text { repetitions of virtual } \\
\text { reality }(V R) \text { shoulder } \\
\text { arthroscopy pro- } \\
\text { gramme }\end{array}$ & $\begin{array}{l}\text { Task completion } \\
\text { time }\end{array}$ & $\begin{array}{l}\text { Performance of } \\
\text { shoulder arthroscopy } \\
\text { task on human cadaver; } \\
\text { no pre-intervention } \\
\text { measurement }\end{array}$ & $\begin{array}{l}\text { Strong correlation between } \\
\text { task completion time on VR } \\
\text { programme and on cadaver; } \\
\text { task completion time on VR } \\
\text { programme significant } \\
\text { predictor of task completion } \\
\text { time on cadaver }\end{array}$ & No \\
\hline $\begin{array}{l}\text { Mehrabi } \\
\text { et al, } 2006^{42}\end{array}$ & $\begin{array}{l}\text { Cohort study; } \\
\text { prospective; } \\
\text { assessors } \\
\text { calculating } \\
\text { performance } \\
\text { score (PS) } \\
\text { blinded to } \\
\text { participants } \\
\text { name and } \\
\text { experience } \\
\text { level }\end{array}$ & $\begin{array}{l}4 \text { Surgeons } \\
\text { (including } 2 \\
\text { trainees) - } 1 \\
\text { Intern; } 1 \\
\text { Resident; } 1 \\
\text { Fellow and } 1 \\
\text { Attending } \\
\text { Surgeon }\end{array}$ & $\begin{array}{l}16 \text { consecutive } \\
\text { operations on rat } \\
\text { model using the Da } \\
\text { Vinci robotic system }\end{array}$ & $\begin{array}{l}\text { Task completion } \\
\text { time, number of } \\
\text { complications } \\
\text { and global PS }\end{array}$ & $\begin{array}{l}4 \text { operations on } \\
\text { anaesthetised pig using } \\
\text { the Da Vinci robotic } \\
\text { system, performed } \\
\text { before and after } \\
\text { intervention }\end{array}$ & $\begin{array}{l}\text { Task completion time } \\
\text { significantly lower in } 3 \text { out of } \\
4 \text { operations post- } \\
\text { intervention; median no. of } \\
\text { complications significantly } \\
\text { lower and PS significantly } \\
\text { higher in all } 4 \text { operations } \\
\text { post-intervention }\end{array}$ & No \\
\hline $\begin{array}{l}\text { Moles et al, } \\
2009^{43}\end{array}$ & $\begin{array}{l}\text { Cohort study; } \\
\text { prospective; } \\
\text { no evidence } \\
\text { of randomisa- } \\
\text { tion }\end{array}$ & $\begin{array}{l}7 \text { Surgical } \\
\text { Residents, all } \\
\text { PGY2 to } 5\end{array}$ & $\begin{array}{l}5 \text { technical tasks } \\
\text { repeated } 3 \text { times on } \\
\text { an inanimate model } \\
\text { using the Da Vinci } \\
\text { robotic system }\end{array}$ & $\begin{array}{l}\text { Task completion } \\
\text { time, number of } \\
\text { errors, severity } \\
\text { of errors; } \\
\text { composite } \\
\text { performance } \\
\text { score (PS) } \\
\text { based on the } \\
\text { three parameters } \\
\text { above (lower the } \\
\text { score, the better } \\
\text { the performance) }\end{array}$ & $\begin{array}{l}\text { Each task and its } \\
\text { repetition evaluated for } \\
\text { each participant; no } \\
\text { pre-intervention } \\
\text { measurements }\end{array}$ & $\begin{array}{l}\text { Mean task completion time } \\
\text { decreased from } 1^{\text {st }} \text { to } 2^{\text {nd }} \\
\text { and from } 2^{\text {nd }} \text { to } 3^{\text {rd }} \text { trial, not } \\
\text { significant; mean number of } \\
\text { errors decreased from trial } \\
\text { to trial, not significant; } \\
\text { composite score significantly } \\
\text { decreased from trial to trial }\end{array}$ & No \\
\hline $\begin{array}{l}\text { Finan et al, } \\
2010^{44}\end{array}$ & $\begin{array}{l}\text { Cohort study; } \\
\text { prospective; } \\
\text { no evidence } \\
\text { of randomisa- } \\
\text { tion }\end{array}$ & $\begin{array}{l}16 \text { Obstetrics } \\
\text { \& Gynaecol- } \\
\text { ogy Resi- } \\
\text { dents, } 3 \\
\text { PGY2, } 7 \\
\text { PGY3, } \\
4 \text { PGY4 }\end{array}$ & $\begin{array}{l}\text { Completion of } \\
\text { training course over } \\
12 \text { months, } 3-4 \text { hrs } \\
\text { each session, } \\
\text { completing } 5 \text { surgical } \\
\text { procedures on an } \\
\text { inanimate model } \\
\text { using the Da Vinci } \\
\text { robotic system }\end{array}$ & $\begin{array}{l}\text { Number of } \\
\text { complications }\end{array}$ & $\begin{array}{l}\text { Evaluation of complica- } \\
\text { tion rate on transfer to } \\
\text { real patients; no pre- } \\
\text { intervention measure- } \\
\text { ments }\end{array}$ & $\begin{array}{l}\text { No complications attributa- } \\
\text { ble to resident training } \\
\text { observed }\end{array}$ & $\begin{array}{l}\text { Yes - } 10 \text { out of } \\
16 \text { participants } \\
\text { evaluated on } \\
\text { part of or whole } \\
\text { operative } \\
\text { procedure on } \\
\text { real patients }\end{array}$ \\
\hline
\end{tabular}


This $3 \mathrm{D}$ environment is usually displayed on a computer screen, with the trainee interacting via a computer interface consisting of modified surgical instruments. The simulated environment allows the practice of particular technical exercises, component parts of a particular procedure, or the completion of entire operative procedures e.g. laparoscopic cholecystectomy (key-hole excision of the gallbladder). The completion of an entire operation is also known as "procedural simulation". VR simulators are also able to provide haptic "force feedback", where the operating surgeon experiences force, motion and vibration through the surgical instruments being used, as if they were actually touching the patient directly themselves. ${ }^{9,45}$

\section{Animal models}

Two studies met the inclusion criteria that described the use of an animal model in surgical simulation, with one randomised-controlled trial ${ }^{36}$ and 1 cohort study. ${ }^{37}$ They describe two different animal models. The first is the use of animals in vivo (Latin, "within the living"), where part of or an entire operative procedure is performed on the whole, live anaesthetised or freshly-killed animal. This is considered high-fidelity simulation, where although there are differences between animal and human anatomy, the identification and control of intra-operative bleeding, sensitive tissue handling and awareness of spatial relationships all closely mimic the real operative environment. ${ }^{46}$ The second animal model is considered to be lower fidelity than the first, and describes the use of animal tissue ex vivo (Latin, "out of the living"). Here, surgical tasks are simulated on organs or tissue that has been removed completely from the animal, e.g. the use of animal small bowel to practice small bowel anastomosis (the technique of re-joining divided bowel). The practice of surgical simulation procedures in anaesthetised animals in vivo is currently prohibited by law in the United Kingdom, but is permitted in other European countries, as well as the United States and elsewhere. ${ }^{45}$

\section{Human cadavers}

The use of human cadavers (the donation of the human body after death) was described in a total of four studies that met the final inclusion criteria. Only one of these was a randomised-control trial, ${ }^{39}$ the remainder were cohort studies. ${ }^{38,40,41}$ Cadaveric simulation provides a high-fidelity model in which the exact anatomical relationships present in live surgical patients are preserved, with almost identical tissue handling and spatial relationships to that of live surgery. Human cadavers can be used in part or in whole, and a single cadaver can provide the opportunity for more than one trainee to perform more than one procedure or task. In the United Kingdom, the practice of operative procedures on human cadavers by surgeons was made possible with the passing of the Human Tissue Act in $2004 .{ }^{47}$

\section{Robotics}

Three studies describing the use of robotic simulation were included, all of which were cohort studies. ${ }^{42-44}$ Robotic systems in surgery are also known as "telemanipulators", and consist of a "robotic stack." This stack interfaces with the patient, and is controlled by the surgeon via an operating console. The stack itself consists of a varying number of robotic "arms" that hold various surgical instruments - thus it is the robot that performs the operative procedure, under the surgeons' control. Advantages of robotic systems include the ability to project a stable, tremor-free 3dimensional operative image; use of tremor-free instruments with 7 degrees of freedom of movement and the ability to experience haptic "force feedback" ${ }^{48}$ Robotic systems are used in surgical simulation to directly improve robotic surgical skills; the robotic system is used by the trainee to perform simulated exercises on either an animal model or an inanimate bench model. The commonest robotic system in clinical use is the da VinciTM robot (Intuitive Surgical, California, USA).

\section{Discussion}

This review demonstrates the benefits of surgical simulation in the development of technical competence. Improvements in outcome measures are demonstrated in every study, across all five main simulation categories. Only one study found such improvements to be independent of simulation training. ${ }^{17}$ These improvements are shown in both the evaluation of technical performance in the simulated environment, and on transfer to the real patient in the operating room. The exception to this is the use of animal models - neither of the two studies included here attempted to demonstrate transfer of simulated skills to the real operating room environment. Where studies compared the use of different simulation techniques, the evidence suggests that the use of bench models and cadaveric simulation is equivalent, ${ }^{39}$ as is the use of bench models and live animals. ${ }^{36}$ Skills learnt on VR and box trainers were also shown to be transferable between the two techniques, with VR simulation providing a greater improvement in the real operating room..$^{22}$

Although an improvement can be seen with the use of each type of simulation technique, several important issues are raised by the various study designs and methodologies, and the variable quality of the research. The first of these is the outcome measures that are used. Over two-thirds of the studies reviewed here use task completion time as a marker of technical competence. Operative speed has been shown to be an objective measurement of technical skill. ${ }^{49}$ However, the time required to complete an operation has many variables, including factors that lie outside of the surgeons' control (e.g. patient factors such as the severity of the disease process, and variable anatomy). The ability to operate quickly does not always equate to the ability to operate safely, and it has therefore been argued that 
although it shows some objectivity, operating speed is a crude measure of skill. ${ }^{50}$ Where studies use only operative speed, trainees and trainers should be wary of accepting such evidence as proof of the effectiveness of simulation.

The issue of operative safety is addressed in many of the studies, with the use of outcome measures that calculate error scores and complication rates. On transfer to the real patient, it can be hypothesized that a reduction in error scores would lead to an improvement in patient outcome. A recent systematic review of technology-enhanced simulation for health professions learners included a total of 609 studies, but only 32 of these studies reported effects on patient care, with a moderate pooled effect. ${ }^{51}$ This highlights a significant difficulty in simulation research. The ultimate purpose of simulation is to develop and improve skills that will be transferred to the real operating room, with the end result being the safe completion of an operation that has improved the patients' health. However, the use of patient outcomes as an outcome measure has significant limitations - first, patient outcomes are affected by many variables that lie outside of the surgeons' control, much like operation speed. Secondly, there is an "ethical imperative" that the supervised trainee performs to the same standard as the supervising "expert" in terms of patient outcomes, regardless of the level of that trainees' skill or experience - if this were not the case, trainees would not be allowed to operate at all. ${ }^{34}$ This "ethical imperative" will always exist in surgical practice, and those involved in both the use of, and research in to, surgical simulation must be aware of this limitation.

Other outcome measures used in several of the studies are the task-specific checklist and the global assessment score. Both of these measures have been shown to be reliable, valid and objective measures of technical skill. ${ }^{52,53}$ Of the two rating scales, however, it has been suggested that the global assessment score is the more reliable. ${ }^{54}$ The use of task-specific checklists, and other task or procedure specific outcomes also poses difficulties in the generalisation of results - simulation that improves a task specific checklist for a laparoscopic gallbladder operation can be generalised to trainees performing that particular procedure, but could not be generalised to a cardiac surgeon performing a valve repair, or an orthopaedic surgeon performing a hip replacement, as the task checklist would have little relevance. It should also be noted that all of the studies described in the "Robotics" category are in fact task-specific to robotic surgery - these simulation studies all use the robotic stack, with a skill-set that is tailored to robotics. Although these studies demonstrate an improvement in technical competence, their results and conclusions should therefore not be generalised beyond the scope of robotics and further research to identify whether robotic skills are transferable to other arenas is necessary.

The transfer of skills between different simulation tools is addressed by a small number of studies, suggesting that skills can be transferred from VR to the human cadaver, ${ }^{41}$ and from the box trainer to VR and vice versa. ${ }^{22}$ In addition, in those studies that attempt to demonstrate transfer of skills from the simulated environment to the real patient (13 studies in total), all but one showed simulation to be effective on transfer. However, it has also been demonstrated elsewhere that specific skill sets in surgery need specific targeted training - Figert et $\mathrm{al}^{55}$ showed that surgeons with considerable experience of open surgery but limited laparoscopic experience were not able to transfer their open surgery experience to newly-acquired laparoscopic skills. Many of the studies reviewed here evaluate laparoscopic skills and laparoscopic procedures. Generalising the results of these studies to non-laparoscopic skills and procedures, and across surgical specialties that use little or no laparoscopic techniques should therefore be attempted with caution.

The heterogeneity of the outcome measures used has been highlighted in previous work on the quality of surgical simulation research. ${ }^{56}$ The same authors also found that studies in surgical simulation often had small participant numbers and lacked statistical power calculations to support their sample sizes. As well as disparate outcome measures, they also commented on the disparate simulation interventions themselves. The review detailed here supports some of these findings. The largest two studies included 50 participants, but a full 27 studies included less than half of this number, with 7 studies having 10 participants or less the smallest study size had only 3 participants. ${ }^{40}$

A statistical power calculation was only found in 2 studies. $^{21,34}$ There is also little uniformity in the simulation exercises, the simulation equipment (e.g. different VR systems and software) or the frequency with which the simulation exercises are performed and practiced. Maargard et $\mathrm{al}^{57}$ have demonstrated that without continual training, skills learnt on a VR simulator were retained at 6 months, but deteriorated between 6 and 18 months. Therefore the issue of simulation frequency highlights an area for further research, to address whether simulation has a lasting effect or whether skills learnt decay over time, and how often trainees should undergo simulation training in order to keep up their skills.

A further feature of the disparate nature of the simulation techniques being reviewed here is the use of "simulation plus..." i.e. the use of simulation as part of a "skills curriculum", accompanied by structured, mentored feedback and instruction, or used in addition to traditional operating room training. Those studies that describe a "skills curriculum" combine simulation with a mixture of demonstration videos, didactic instruction, lectures, procedural demonstrations by experts and/or written material. However, no attempt is made to separate the effect of the simulation exercises from these additional teaching modalities. Therefore, whilst the benefit of these curricula can clearly be seen, the precise contribution of their individual components is less so. These difficulties are com- 
pounded by the fact that no two "skills curriculae" described in these simulation studies are exactly alike.

The precise influence of instruction and feedback during simulation practice is also unclear in most of the studies, as once again it is not separated from the simulation exercise itself. The exception is the study by Risucci et $\mathrm{al}^{16}$, who specifically set out to determine the effect of simulation practice and additional instruction on laparoscopic skills. This additional instruction takes the form of a demonstration video and tutor feedback. Those undergoing simulation plus instruction showed an improvement in task completion times, plus a greater improvement in both error rate and in variability of performance. This suggests that simulation practice is augmented by instruction and feedback from an expert tutor. The influence of feedback is not unnoticed in the wider educational literature. Feedback is seen as an integral part of Kolb's experiential learning cycle, where the learners' ideas are formed and modified through experience.$^{58}$ Hill argues that feedback plays an important role in Kolb's cycle as it supports the process of reflection and the consideration of new and more in-depth theories, and helps the learner plan more productively for their next learning experience. ${ }^{59}$

Improving classroom learning through the use of assessment has also been shown to be dependent on the provision of feedback to learners, in order to help them recognise both the standards they are aiming for and the next steps they need to take in the learning process. ${ }^{60}$ The lack of emphasis on feedback in surgical simulation studies may be because such feedback from expert tutors must come at an additional cost in both man-power and time that is potentially difficult to meet.

The additional effect of instruction and feedback also suggests that simple "quantity" and repetition of simulation exercises is not sufficient; rather, it is both the "quantity" and the "quality" of simulation that is important. This is borne out by Joyce et $\mathrm{al}^{20}$ who showed that although simulation improved performance, no correlation was found between the amount of time spent practising and task completion time, with only a low correlation between practice time and technical skill. These findings on "quality vs quantity" are supported by Ericsson et al's theory of "deliberate practice." They suggest that simply having sufficient experience or undergoing a sufficient amount of practice is not enough for the achievement of maximal performance; rather, it is the precise nature of the practice itself that leads to maximal performance.

They define "deliberate practice" as "activities that have been specifically designed to improve the current level or performance," and propose that deliberate practice must take into account the learners motivation and their preexisting knowledge, must be accompanied by immediate formative feedback, and should extend over a period of at least 10 years in order for "expert performance" to be achieved. ${ }^{61}$

\section{Limitations of the study}

The literature on simulation in surgery is international, with a significant amount of work from the US. In order to compare such international research, the notion of a "generic level" of basic clinical competence before embarking on surgical training is therefore an assumption - that surgical trainees across differing countries all achieve the same basic level of competence before their surgical training begins. In evaluating the literature on the use of surgical simulation across different surgical specialties, another assumption is made, that the technical skills and tasks practiced repeatedly in one specialty are generic and transferable between all specialties. A further inherent limitation to the use of a literature-based methodology is that the conclusions of the review rely heavily on the methodological adequacies, or inadequacies, of the studies included; such conclusions must therefore take into account the quality and rigour of the research being reviewed. This review has also been conducted by a single author, who was solely responsible for the selection of the included studies and for data analysis; this introduces the potential for bias.

\section{Conclusions}

- Five main categories of simulation technique currently used to develop technical competence in surgical training are identified here - the use of bench models and box trainers; Virtual Reality; human cadavers; animal models and robotics. On reviewing the available evidence, the benefits of all five of these techniques in improving technical skills can be seen within the simulated environment. All but the use of animal models show the ability to transfer skills to the real patient in the operating room environment. Therefore surgical trainees should be confident in the effects of using simulation during their training, and those involved in the planning of surgical training should endeavour to provide trainees with access to formal, structured simulation.

- When considering the evidence for surgical simulation, both trainees and trainers should be aware of the taskspecific nature of surgical simulation research. As a consequence, trainees should tailor their simulation training to those simulation exercises designed to improve the skills that form a significant part of their daily practice (e.g. laparoscopic exercises for those that practice laparoscopic techniques). When designing surgical training, educational leaders must also be aware that surgical simulation needs to be tailored to the needs of individual trainees in individual specialties.

- The use of cadaveric simulation is equivalent to the use of VR or box trainers. Due to the scarcity of cadaveric material, and the ethical and moral issues around its use, resources should be directed towards training on VR and box trainers.

- Only a very small number of studies detail the use of animal models in surgical trainees, and they did not at- 
tempt to demonstrate transfer of skills to the real patient. The use of a bench model was also shown to be equivalent to the use of live animals. Therefore, as animal models also carry significant ethical and moral issues, and the use of live animals is prohibited in the UK, other methods of simulation should be considered when planning surgical training.

- Skills learnt on both box trainers and VR are transferable to the real patient, with the evidence suggesting the slight superiority of VR. However, VR equipment is more expensive. Surgical skills curricula should therefore incorporate simulation on box trainers, with VR being used in addition, where resources allow.

- Simulation on robotic systems has a direct effect on the development of robotic skills, but whether such skills transfer to other surgical arenas is unknown. With the cost of robotic systems so high, robotic simulation should only be considered in those trainees who will definitely need to use robotics in their daily practice; the number of such trainees is limited at present due to the very small number of centres utilising robotics.

- To enhance the benefits of structured simulation, trainers should provide time for trainees to receive expert instruction and feedback during their simulation training. Such feedback should be delivered in additional to self-directed practice.

- Areas for future research in surgical simulation include the determination of how skills learnt during simulation exercises are retained, the frequency and intensity of simulation that provides the maximum benefit, and further work on the transfer of skills between different simulation techniques, particularly the transfer of robotic skills. The complex nature of educational interventions must also be recognised by those planning and evaluating surgical simulation research, particularly when designing a "skills curriculum".

\section{Conflict of Interest}

The author declares that he has no conflict of interest.

\section{References}

1. Kneebone RL, Scott W, Darzi A, Horrocks M. Simulation and clinical practice: strengthening the relationship. Med Educ. 2004;38:1095-1102.

2. Department of Health. The European Working Time Directive-UK notification of derogation for doctors in training. 2009[cited 15 August 2012]; Available from: http://www.dh.gov.uk/prod_consum_dh/groups/dh_ digitalassets/documents/digitalasset/dh_093943.pdf.

3. Chikwe J, de Souza AC, Paper JR. No time to train the surgeons. BMJ. 2004;328:418-419.

4. Crofts TJ, Griffiths JM, Sharma S, Wygrala J, Aitkin RJ. Surgical training: an objective assessment of recent changes for a single health board. BMJ. 1997;314:814.

5. Bradley P. The history of simulation in medical education and possible future directions. Med Educ.2006;40:254-262.

6. Ziv, A, Wolpe, PR, Small, S, Glick, S. Simulation-based medical education: an ethical imperative. Acad Med.2003;78:783-788.

7. Kunkler, K. The role of medical simulation: an overview. Int J Med Robot. 2006;2:203-210.
8. Bell RH. Why Johnny cannot operate. Surgery. 2009;146:533-542.

9. Hammoud MM, Nuthalapaty FS, Goepfert AR, Casey PM, Emmons S, Espey, EL et al. To the point: medical education review of the role of simulators in surgical training. Am J Obstet Gynecol. 2008;199:338-343.

10. Norman G, Dore K, Grierson L. The minimal relationship between simulation fidelityand transfer of learning. Med Educ. 2012;46:636-647.

11. Murphy JG, Cremonin F, Kane GC, Dunn W. Is simulation based medicine training the future of clinical medicine? Eur Rev Med Pharmacol Sci. 2007;11:1-8.

12. Issenberg SB, McGaghie WC, Petrusa ER, Gordon DL, Scalese RJ. Features and uses of high-fidelity medical simulations that lead to effective learning: a BEME systematic review. Med Teach. 2005;27:10-28.

13. Derossis AM, Bothwell J, Sigman HH, Fried GM. The effect of practice on performance in a laparoscopic simulator. Surg Endosc.1998;12:11171120 .

14. Scott DJ, Bergen PC, Rege RV, Laycock R, Tesfay ST, Valentine RJ et al. Laparoscopic training on bench models: better and more cost effective than operating room experience? J Am Coll Surg. 2000;191:272-283.

15. Hamilton EC, Scott DJ, Kapoor A, Nwariaku F, Bergen PC, Rege RV et al. Improving operative performance using a laparoscopic hernia simulator. Am J Surg. 2001;182:725-728.

16. Risucci D, Cohen JA, Garbus JE, Goldstein M, Cohen MG. The effects of practice and instruction on speed and accuracy during resident acquisition of simulated laparoscopic skills. Curr Surg. 2001;58:230-235.

17. Traxer O, Gettman MT, Napper CA, Scott DJ, Jones DB, Roehrborn CG et al. The impact of intense laparoscopic skills training on the operative performance of urology residents. J Urol. 2001;166:1658-1661.

18. Korndorffer JR, Dunne JB, Sierra R, Stefanidis D, Touchard CL, Scott DJ. Simulator training for laparoscopic suturing using performance goals translates to the operating room. J Am Coll Surg. 2005;201:23-29.

19. Banks EH, Chudnoff S, Karmin I, Wang C, Pardanani S. Does a surgical simulator improve resident operative performance of laparoscopic tubal ligation? Am J Obstet Gynecol. 2007;197:e1-e5.

20. Joyce DL, Dhillon TS, Caffarelli AD, Joyce DD, Tsirigotis DN, Burdon TA et al. Simulation and skills training in mitral valve surgery. $J$ Thorac Cardiovasc Surg. 2011;141:107-112.

21. Price J, Naik V, Boodhwani M, Brandys T, Hendry P, Lam BK. A randomised evaluation of simulation training on performance of vascular anastomosis on a high-fidelity in vivo model: the role of deliberate practice. J Thorac Cardiovasc Surg. 2011;142:496-503.

22. Hamilton EC, Scott DJ, Fleming JB, Rege RV, Laycock R, Bergen PC et al. Comparison of video trainer and virtual reality training systems on acquisition of laparoscopic skills. Surg Endosc. 2002;16:406-411.

23. Seymour NE, Gallagher AG, Roman SA, O’Brien MK, Bansal VK, Andersen DK et al. Virtual reality training improves operating room performance - results of a randomised, double-blinded study. Ann Surg. 2002;236:458-464.

24. Grantcharov TP, Kristiansen VB, Bendix J, Bardam L, Rosenberg J, Funch-Jensen, P. Randomised clinical trial of virtual reality simulation for laparoscopic skills training. BMJ. 2004;91:146-150.

25. Dayal R, Faries PL, Lin SC, Bernheim J, Hollenbeck S, DeRubertis B et al. Computer simulation as a component of catheter-based training. J Vasc Surg. 2004;40:1112-1117.

26. McClusky III DA, Gallagher AG, Ritter EM, Lederman AB, Van Sickle $\mathrm{KR}$, Baghai $\mathrm{M}$ et al. Virtual reality training improves junior residents' operating room performance: results of a prospective, randomised, doubleblinded study of the complete laparoscopic cholecystectomy. J Am Coll Surg. 2004;199:S73.

27. Andreatta PB, Woodrum DT, Birkmeyer JD, Yellamanchilli RK, Doherty GM, Gauger PG et al. Laparoscopic skills are improved with LapMentorTM training - results of a randomised, double-blinded study. Ann Surg. 2006;243:854-863.

28. Chaer RA, DeRubertis BG, Lin SC, Bush HL, Karwowski JK, Birk D et al. Simulation improves resident performance in catheter-based intervention results of a randomised, controlled study. Ann Surg. 2006;244:343-352.

29. Ahlberg G, Enochsson L, Gallagher AG, Hedman L, Hogman C, McClusky III DA et al. Proficiency-based virtual reality training significantly reduces the error rate for residents during their first 10 laparoscopic cholecystectomies. Am J Surg. 2007;193:797-804. 
30. Cosman PH, Hugh TJ, Shearer CJ, Merrett ND, Biankin AV, Cartmill JA. Skills acquired on virtual reality laparoscopic simulators transfer into the operating room in a blinded, randomised, controlled trial. Stud Health Technol Inform. 2007;125:76-81.

31. Dawson DL, Meyer J, Lee ES, Pevec WC. Training with simulation improves residents' endovascular procedure skills. J Vasc Surg. 2007;45:149154.

32. Balasundaram I, Aggarwal R, Darzi A. Short-phase training on a virtual reality simulator improves technical performance in tele-robotic surgery. Int J Med Robot. 2008;4:139-145.

33. Verdaasdonk EGG, Dankelman J, Lange JF, Stassen LPS. Transfer validity of laparoscopic knot-tying training on a VR simulator to a realistic environment: a randomised controlled trial. Surg Endosc. 2008;22:16361642 .

34. Larsen CR, Soerensen JL, Grantcharov TP, Dalsgaard T, Schouenborg L, Ottosen $\mathrm{C}$ et al. Effect of virtual reality training on laparoscopic surgery: randomised control trial. BMJ. 2009;338:b1802.

35. Maschuw K, Schlosser K, Kupietz E, Slater EP, Weyers P, Haasan I. Do soft skills predict surgical performance? A single-center randomised controlled trial evaluating predictors of skill acquisition in virtual reality laparoscopy. World J Surg. 2011;35:480-486.

36. Fried GM, Derossis AM, Bothwell J, Sigman HH. Comparison of laparoscopic performance in vivo with performance measured in a laparoscopic simulator. Surg Endosc. 1999;13:1077-1081.

37. Bijoy Thomas M, Dandolu V, Caputo P, Milner R, Hernandez E. Resident education in principles and technique of bowel surgery using an ex-vivo porcine model. Obstet Gynecol Int. 2010; 2010:852647.

38. Martin M, Vashist B, Frezza E, Ferone T, Lopez B, Pahuja M et al. Competency-based instruction in critical invasive skills improves both resident performance and patient safety. Surgery. 1998;124:313-317.

39. Anastakis DJ, Regehr G, Reznick RK, Cusimano M, Murnaghan J, Brown $\mathrm{M}$ et al. Assessment of technical skills transfer from the bench training model to the human model. Am J Surg. 1999;177:167-170.

40. Bergeson RK, Schwend RM, DeLucia T, Silva SR, Smith JE, Avilucea FR. How accurately do novice surgeons place thoracic pedicle screws with the free hand technique? Spine. 2008;33:E510-E507.

41. Martin KD, Belmont PJ, Schoenfeld AJ, Todd M, Cameron KL, Owens BD. Arthroscopic basic task performance in shoulder simulator model correlates with similar task performance in cadavers. J Bone Joint Surg. 2011;93:e127(1-5).

42. Mehrabi A, Yetimoglu CL, Nickkholgh A, Kashfi A, Kienle P, Kostantinides $\mathrm{L}$ et al. Development and evaluation of a training module for the clinical introduction of the da Vinci robotic system in visceral and vascular surgery. Surg Endosc. 2006;20:1376-1382.

43. Moles JJ, Connelly PE, Sarti EE, Baredes S. Establishing a training program for residents in robotic surgery. Laryngoscope. 2009;119:1927-1931.
44. Finan MA, Clark ME, Rocconi RP. A novel method for training residents in robotic hysterectomy. J Robot Surg. 2010;4:33-39.

45. Sarker SK, Patel B. Simulation and surgical training. Int J Clin Pract. 2007;61:2120-2125.

46. Wolfe BM, Szabo Z, Moran ME, Chan P, Hunter JG, Training for minimally invasive surgery - need for surgical skills. Surg Endosc. 1993;7:93-95.

47. Gilbody J, Prasthofer AW, Ho K, Costa ML. The use and effectiveness of cadaveric workshops in higher surgical training: a systematic review. Ann R Coll Surg Engl. 2011;93:347-352.

48. Jayne DG, Culmer PR, Barrie J, Hewson R, Neville A. Robotic platforms for general and colorectal surgery. Colorectal Dis. 2011;13(S7):78-82.

49. van Rij AM, McDonald JR, Pettigrew RA, Putterill MJ, Reddy CK, Wright JJAD. Cusum as an aid to early assessment of the surgical trainee. $\mathrm{Br}$ J Surg. 1995;82:1500-1503.

50. Darzi A, Smith S, Taffinder N. Assessing operative skill - needs to become more objective. BMJ. 1999;318:887-888.

51. Cook DA, Hatala R, Brydges R, Zendejas B, Szostek JH, Wang AT et al. Technology-enhanced simulation for health professions education. JAMA. 2011;306:978-988.

52. Martin JA, Regehr G, Reznick R, MacRae H, Murnaghan J, Hutchinson $\mathrm{C}$ et al. Objective structured assessment of technical skill (OSATS) for surgical residents. Br J Surg. 1997;84:273-278.

53. Reznick R, Regehr G, MacRae H, Martin J, McCulloch W. Testing technical skill via an innovative "bench station" examination. Am J Surg. 1997;172:226-230.

54. Regehr G, MacRae H, Reznick RK, Szalay D. Comparing the psychometric properties of checklists and global rating scales for assessing performance on an OSCE-format examination. Acad Med. 1998;73:993-997.

55. Figert PL, Park AE, Witzke DB, Schartz RW. Transfer of training in acquiring laparoscopic skills. J Am Coll Surg. 2001;193:533-537.

56. Sutherland LM, Middleton PF, Anthony A, Hamdorf J, Cregan P, Scott D et al. Surgical simulation - a systematic review. Ann Surg. 2006;243 291-300.

57. Maargard M, Sorensen JL, Ostergaard J, Dalsgaard T, Grantcharov TP, Ottesen BS et al. Retention of laparoscopic procedural skills acquired on a virtual-reality surgical trainer. Surg Endosc. 2011;25:722-727.

58. Kolb DA. Experiential learning: experience as the source of learning and development. New Jersey: Prentice Hall; 1984.

59. Hill F. Feedback to enhance student learning: facilitating interactive feedback on clinical skills. IJOCS. 2007;1:21-24.

60. Black P, Wiliam D. Assessment and classroom learning. Assessment in education. 1998;5:7-74.

61. Ericsson K, Krampe R, Tesch-Römer C. The role of deliberate practice in the acquisition of expert performance. Psychol Rev. 1993;100:363-406. 